CLINICAL STUDY

\title{
Automated image analysis of hand radiographs reveals widened joint spaces in patients with long-term control of acromegaly: relation to disease activity and symptoms
}

\author{
N R Biermasz ${ }^{1}$, R van 't Klooster ${ }^{2}$, M J E Wassenaar ${ }^{1}$, S H Malm ${ }^{1}$, K M J A Claessen ${ }^{1}$, R G H H Nelissen ${ }^{3}$, \\ F Roelfsema $^{1}$, A M Pereira ${ }^{1}$, H M Kroon ${ }^{4}$, B C Stoel ${ }^{2}$, J A Romijn ${ }^{1}$ and M Kloppenburg ${ }^{5}$ \\ ${ }^{1}$ Departments of Endocrinology and Metabolism C4-R, ${ }^{2}$ Division of Image Processing, Department of Radiology, ${ }^{3}$ Department of Orthopedics, ${ }^{4}$ Department \\ of Radiology and ${ }^{5}$ Department of Rheumatology, Leiden University Medical Center, PO Box 9600, 2300 RC Leiden, The Netherlands
}

(Correspondence should be addressed to K M J A Claessen; Email: k.m.j.a.claessen@lumc.nl)

\begin{abstract}
Objective: Arthropathy is an invalidating complication of acromegaly. Although acromegalic arthropathy shares features with primary osteoarthritis, joint spaces are widened rather than narrowed in patients with long-term cure of acromegaly. The late effects of acromegaly on hand joints have not been characterized. Therefore, the objective of the current study was to assess joint space widths (JSWs) of hand joints in patients with long-term control of acromegaly and to identify factors associated with JSW.

Methods: A cross-sectional study was carried out in 89 patients (age $58 \pm 12$ years, $49 \%$ women) with long-term controlled acromegaly and 471 controls without hand symptoms (age $46 \pm 12$ years, $42 \%$ women). Radiological JSWs of individual hand joints were measured by automated image analysis. Results: Patients had wider mean joint spaces than controls: metacarpo-phalangeal (MCP) joints were $\sim 24 \%$, proximal interphalangeal joints $\sim 21 \%$, and distal interphalangeal joints were $\sim 20 \%$ wider (patients vs controls; $P<0.001$ for all joints). Mean JSW exceeded the 95th percentile of the values obtained in controls in $64 \%$ of patients. Higher IGF1 and GH concentrations at diagnosis were associated with larger JSWs (adjusted $\beta$ for pretreatment GH in tertiles: 0.09 (95\% confidence interval (CI) 0.03-1.84) and for IGF1 in tertiles: 0.14 (95\% CI 0.05-0.23) at the MCP joints in acromegalic patients. In male patients, but not in female patients, increased JSWs were associated with more self-reported pain $(P=0.02)$.

Conclusions: Using a new semi-automated image analysis of hand radiographs, acromegalic patients with long-term disease control appeared to have increased joint spaces of all hand joints. JSWs were positively related to disease activity at diagnosis, but not to duration of follow-up, suggesting irreversible cartilage hypertrophy. Irreversible cartilage hypertrophy may partly explain persisting hand complaints despite long-term disease control.
\end{abstract}

European Journal of Endocrinology 166 407-413

\section{Introduction}

Acromegaly is a rare disease caused by overproduction of GH from pituitary adenomas (1). It is characterized by a variety of complaints, and increased co-morbidity and mortality. On radiographs, active acromegaly is characterized by increased joint space widths (JSWs) (2). However, the effects of long-term cure or biochemical control of acromegaly on JSW have not been established. Nonetheless, patients with acromegaly have a high prevalence of joint pain and arthropathy even after long-term biochemical cure (3). Although the changes in joints of patients with controlled acromegaly match the criteria of both clinical and radiological osteoarthritis (OA) (4), characteristics of radiological OA differ between controlled acromegaly and primary OA: patients with acromegaly have more severe osteophytosis, but less joint space narrowing (4). In addition, the joint spaces in acromegaly patients appear to remain abnormally wide, reflecting persistent cartilage hypertrophy despite long-term biochemical control of acromegaly.

At present, there are no quantitative data available on the magnitude of JSWs in patients with acromegaly. Therefore, the aim of the present study was to quantify JSWs in hand radiographs of patients with long-term control of acromegaly in comparison with age- and gender-matched controls. We used semi-automated image analysis software (5) for this purpose. The second aim was to explore the potential factors that are 
associated with the size of the joint space in acromegalic patients, such as age at diagnosis, markers of severity, duration of acromegaly, and duration of remission. The third aim was to explore the relation between JSWs and specific joint complaints in these patients with controlled acromegaly.

\section{Materials and methods}

\section{Patients}

We compared JSW of hand joints in patients with longterm control of acromegaly with that of control subjects.

Acromegalic patients All patients with long-term remission of acromegaly (i.e. more than 2 years), according to a normal insulin-like growth factor 1 (IGF1) concentration for age, were selected from our database. The Leiden Acromegaly Database includes baseline and follow-up results of all patients who have been treated in the Leiden University Medical Center, a tertiary referral center with dedicated pituitary surgeons, from 1977 onward. We invited 126 patients to participate in the present cross-sectional study, of whom $89(71 \%)$ agreed to participate.

The majority of the patients were treated by primary transsphenoidal surgery, if necessary, followed by additional treatment. Control of GH oversecretion was achieved by curative transsphenoidal surgery $(n=49)$, surgery and radiotherapy $(n=15)$, primary surgery followed by somatostatin analog treatment $(n=15)$, all three modes of treatment $(n=3)$, and primary somatostatin analog treatment $(n=7)$. At the study visit, all patients were in biochemical remission for a mean of 14 years. Biochemical remission was defined as normal glucose, suppressed serum GH concentrations below $0.38 \mu \mathrm{g} / \mathrm{l}$ (in patients not treated with somatostatin analogs), serum GH concentrations below $1.9 \mu \mathrm{g} / \mathrm{l}$, and normal IGF1 levels for age at yearly follow-up visits (6). Hypopituitarism was defined by the presence of clinically relevant hormonal deficiencies in minimal one pituitary axis and was supplemented with thyroxine $\left(\mathrm{T}_{4}\right)$, hydrocortisone, testosterone, or estrogens (in premenopausal women) according to the following definitions. Estrogen deficiency in women was present in the case of LH/FSH deficiency in premenopausal women with prolonged amenorrhea $(>1$ year) without adequate replacement therapy or with a low serum estradiol concentration of $<70 \mathrm{nmol} / \mathrm{l}$ and in all postmenopausal women. In men, LH/FSH deficiency was defined as testosterone level below the reference range $(8.0 \mathrm{nmol} / \mathrm{l})$. Thyroid-stimulating hormone (TSH) deficiency was defined as a thyroxine (free $\mathrm{T}_{4}$ ) level below the reference range (absolute value $<10 \mathrm{pmol} / \mathrm{l}$ ). ACTH deficiency was defined as an insufficient increase in cortisol levels (absolute value $<0.55 \mu \mathrm{mol} / \mathrm{l}$ ) after ACTH test or insulin tolerance test. $\mathrm{GH}$ deficiency was not routinely assessed in all patients.
Controls An age- and gender-matched control group was derived from databases of The Leiden Early Arthritis Clinic (EAC, $n=167$ ) (7) and a prospective study in patients with knee complaints $(n=304)(8)$. None of these controls had hand symptoms. EAC is a prospective study that was started in 1993 and includes patients with early arthritis with symptoms for $<2$ years (7). The goal is to detect inflammatory disorders early in the disease state and to treat these accordingly. All parameters such as medical history and physical diagnostics examination were obtained by trained staff consisting of rheumatologists and research nurses. Conventional radiographs of hands, feet and affected joints were obtained in all patients at baseline. For the purpose of the present study, we selected patients without hand symptoms and used the hand radiographs taken at their inclusion visit. The second study is an epidemiological study which includes patients with traumatic or non-traumatic subacute knee complaints (8). At a follow-up visit 10 years later, routine radiographs of the hands were performed in all patients. Since patients were not included in the study on the basis of hand joint pathology, we assumed that their hand joints are a valid sample of the general population.

The protocols of the studies were approved by the Medical Ethics Committee. Written informed consent was given by all patients and controls who participated in the studies.

\section{OA diagnosis}

In all subjects, clinical OA was scored according to the criteria proposed by the American College of Rheumatology (ACR) (9).

\section{Study parameters}

Conventional radiographs of the hands From all subjects with acromegaly and the second control group, standardized digital hand radiographs were taken by the same radiological technician with standardized focusdetector distances. Of the EAC controls, 133 radiographs were analog and 39 were digital. For computerized analysis, the analog radiographs were digitized first (VXR-12, VIDAR System Corporation, Herndon, VA, USA). Hand joints were evaluated for the presence of radiological OA using the Kellgren-Lawrence classification system $(10,11)$. This is a 5-point ranking scale (O, normal OA; 4, severe OA), in which a score of 2 or higher is considered to be (mild) OA.

Image processing for determination of JSW Recently, van 't Klooster et al. (5) developed in our institute a semi-automatic quantitative method to measure JSW in hand radiographs with a good correlation with semi-quantitative scoring of joint space narrowing (Fig. 1). Therefore, JSWs of the hands in the current 


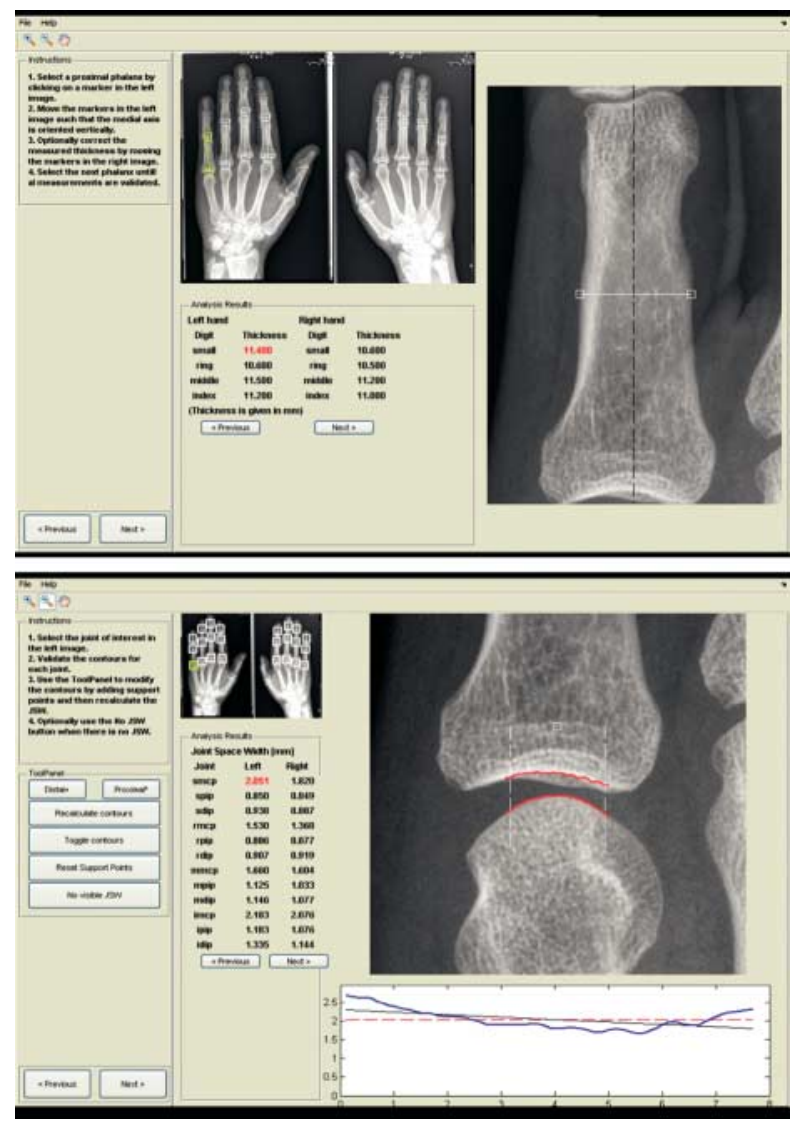

Figure 1 Example of joint space width assessment in a hand joint of a patient with acromegaly.

study were measured using this semi-automatic image analysis program. First, this program loads the digital/digitized (vide supra) radiographs and the user separates the left and the right hand. Subsequently, in each hand, 12 joints, being four distal interphalangeal (DIP), four proximal interphalangeal (PIP), and second to fifth metacarpo-phalangeal (MCP) joints, were analyzed semi-automatically in four steps: i) identification of the joint locations, ii) detection of the proximal and distal margins of each joint, iii) measurement of the width of each proximal phalange, as a reference for the JSW measurement interval, and iv) calculation of the width of the joint space within the determined measurement interval. The automatic results of each step were reviewed by an expert (S H Malm) and corrected if needed. For each subject, 24 measured joint spaces, as well as the thickness of the proximal phalanges, were reported. The joints of the thumb were omitted from the analysis since they were not perpendicular to the imaging plane and could not be assessed reliably. The individual values and the mean results of all joints, the MCP, PIP, and DIP joints were used for analysis. The intra-individual variation between repeat readings $(n=25)$ was low $(<5 \%)$, reflected by a $\kappa$ value of $>0.9$. Within the EAC cohort, we compared digitized analog and digital radiographs. There was no significant difference between both techniques for any of the mean joint spaces $(P=0.30)$.

Pain Self-reported painful joints were recorded on a standard diagram including all 24 hand joints. Pain during structured physical examination was recorded systematically for all 24 joints.

Disease characteristics in acromegaly patients In order to estimate the duration of exposure to previous $\mathrm{GH}$ excess, the estimated disease duration before diagnosis and disease duration before remission were carefully assessed. Estimated onset of disease was based on the start of signs and symptoms and changes on photographs. The date of remission was the date of normalization of GH and IGF1 concentrations. The duration of follow-up was the period between diagnosis and study visit. The duration of remission was the period between normalization of GH/IGF1 concentrations and study visit. At the study visit, the actual GH and IGF1 concentrations were measured. Pretreatment GH and IGF1 concentrations were recorded from the database. Since IGF1 analysis was not available in our center before 1986, pretreatment IGF1 levels were not available for all cases (only 67 of 89 patients).

Assays At the study visit, and after 1993, serum GH level was measured with a sensitive immunofluorometric assay (Wallac, Turku, Finland) specific for the $22 \mathrm{kDa}$ GH protein, calibrated against WHO International Reference Preparation (WHO IRP) 80/505 (detection limit: $0.3 \mu \mathrm{g} / \mathrm{l}$; intra-assay coefficient of variation (CV) $1.6-8.4 \%$ of $0.1-15.4 \mu \mathrm{g} / \mathrm{l}$ ). Before 1992, GH was measured by RIA (Biolab, Serona, Coissins, Switzerland) calibrated against WHO IRP 66/21 (detection limit: $0.5 \mathrm{mU} / \mathrm{l}$, inter-assay $\mathrm{CV}$ $<5 \%$; for the conversion of $\mu \mathrm{g} / \mathrm{l}$ to $\mathrm{mU} / \mathrm{l}$, multiply by 2). From 1986 to 2005, serum IGF1 concentrations were determined by RIA (Incstar, Stillwater, MN, USA) with a detection limit of $1.5 \mathrm{nmol} / \mathrm{l}$ and an inter-assay $\mathrm{CV}<11 \%$. IGF 1 is expressed as SDS for age- and gender-related normal levels determined in the same laboratory (12). From 2005, serum IGF1 concentration $(\mathrm{ng} / \mathrm{ml})$ was measured using an immunometric technique on an Immulite 2500 system (Diagnostic Products Corporation, Los Angeles, CA, USA). The intra-assay variation was 5.0 and $7.5 \%$ at mean plasma levels of 8 and $75 \mathrm{nmol} / \mathrm{l}$ respectively. IGF1 levels were expressed as age- and gender-dependent SDS, using lambda-mu-sigma (LMS) smoothed reference curves based on measurements in 906 healthy individuals $(13,14)$.

\section{Statistical analysis}

For data analysis, PASW 17 for Windows was used (SPSS, Inc., Chicago, IL, USA). We reported the mean 
\pm s.D. unless specified otherwise. For comparison of the mean JSWs between patients and controls, we used ANOVA analysis with adjustments for age and gender. For the correlation between JSW and disease characteristics of acromegaly, we used Pearson's correlation and linear regression analysis. Tertiles of serum GH and IGF1 concentrations and sex were used as fixed factors, and age was used as a covariate. (Un)standardized betas $(\beta)$ were reported with 95\% confidence intervals $(95 \%$ CIs). Mean joint spaces were dichotomized to normal $(<95$ th percentile of female or male controls per joint site) or increased $(>95$ th percentile of female or male controls per joint site).

In addition, we studied self-reported pain in relation to the joint space of the individual joints. Individual JSWs were re-coded in deciles for each joint pair (i.e. left and right MCP 2-5, DIP 2-5, and PIP 2-5; $n=12$ ) per gender within acromegalic patients. The decile score was used as a covariate in a general estimated equation model to take into account the intra-patient effect.

\section{Results}

\section{Baseline characteristics}

The mean age of the 89 included acromegaly patients was $58.2 \pm 11.5$ years and there was an equal gender distribution (49\% females). Mean body mass index (BMI) was $28.5 \pm 4.7 \mathrm{~kg} / \mathrm{m}^{2}$. At the time of diagnosis, the IGF1 SDS was $7.4 \pm 4.7(n=67)$ and the GH concentration was $44.5 \pm 63 \mu \mathrm{g} / \mathrm{l}$. The estimated duration of acromegaly before the moment of diagnosis was $8.8 \pm 7.4$ years. All patients were in biochemical remission of acromegaly and the mean duration of remission was $14 \pm 6$ years. One or more pituitary hormone deficiencies were present in $33 \%$ of patients; ACTH and TSH deficiency was present in 25 and $20 \%$ of patients respectively, and the patients were adequately supplemented with hydrocortisone and $\mathrm{T}_{4}$. LH/FSH deficiency, including natural menopause and hypogonadotropic hypogonadism, was present in $54 \%$ of patients. Sex hormone substitution therapy was given to ten patients (nine men used testosterone and one woman used estrogens). The majority of patients with acromegaly (84\%) reported pain and stiffness of the hand at the time of the current evaluation. The prevalence of clinical hand OA in acromegaly according to the ACR criteria was $40 \%$. The mean age of the 470 controls was $46 \pm 12$ years, which was lower compared with that of the acromegalic patients $(P<0.001)$, and $42 \%$ were females, which was not different from the gender distribution in the patients $(P=0.178)$.

\section{Hand JSW in acromegaly patients and controls}

Both among acromegaly patients and controls, men had wider joint spaces and larger proximal phalange widths than women $(P<0.001)$. There was a negative correlation between JSW and age for the MCP $(R=-0.31, P<0.003)$ and DIP joints in acromegalic patients $(R=-0.22, P<0.04)$, but not for the PIP joints $(R=0.024, P=0.825$ (not significant $(\mathrm{NS}))$ ).

In acromegaly patients, the JSWs of all 24 hand joints were increased compared with those of controls (mean difference adjusted for age and gender was $0.26 \mathrm{~mm}$ (95\% CI 0.22-0.30), $P<0.001$; Table 1, Fig. 2). Mean MCP joint spaces were increased by $24 \%$, mean PIP joint spaces by $21 \%$, and mean DIP joint spaces by $20 \%$ in patients compared with controls (Table 1). The mean width of the proximal phalanx was also increased in acromegaly patients by $7 \%(P<0.001)$.

Mean joint spaces above the 95th percentile of controls were observed in 53\% of the MCP, $45 \%$ of the PIP, $27 \%$ of the DIP joint sites, and in $64 \%$ (all 24 measured joints) of acromegalic patients. Only $9 \%$ of the acromegalic patients had mean JSW (all joints) below the 50th percentile of controls.

\section{Influence of (previous) activity of acromegaly on joint space}

For both MCP and PIP joints, there was a positive correlation between JSWs and pretreatment serum GH and IGF1 concentrations (both $R=0.35, P=0.001$ ). In contrast to the other hand joints, DIP joints were not significantly associated with parameters of severity of acromegaly disease. A negative correlation

Table 1 Patients with acromegaly have wider mean JSWs than controls. All measurements are expressed in millimeters (S.D.).

\begin{tabular}{|c|c|c|c|c|c|}
\hline Mean JSW (mm) & $\begin{array}{c}\text { Acromegalic } \\
\text { patients }(n=89)\end{array}$ & $\begin{array}{l}\text { Controls } \\
(n=470)\end{array}$ & Mean difference & $\begin{array}{c}\text { Adjusted mean } \\
\text { difference }^{\mathrm{a}}\end{array}$ & $\begin{array}{c}\boldsymbol{P}_{\text {value }}{ }^{\mathrm{b}} \\
\text { (two-tailed) }\end{array}$ \\
\hline All joints & $1.38 \pm 0.25$ & $1.15 \pm 0.17$ & $0.23(0.19-0.27)$ & $0.26(0.22-0.30)$ & $<0.001$ \\
\hline MCP & $1.95 \pm 0.34$ & $1.61 \pm 0.23$ & $0.34(0.28-0.40)$ & $0.38(0.33-0.44)$ & $<0.001$ \\
\hline PIP & $1.15 \pm 0.23$ & $0.95 \pm 0.15$ & $0.20(0.16-0.24)$ & $0.20(0.17-0.25)$ & $<0.001$ \\
\hline DIP & $1.04 \pm 0.30$ & $0.89 \pm 1.78$ & $0.14(0.10-0.19)$ & $0.18(0.14-0.23)$ & $<0.001$ \\
\hline $\begin{array}{l}\text { Mean width proximal } \\
\text { phalanx }(\mathrm{mm})\end{array}$ & $10.19 \pm 1.26$ & $9.40 \pm 1.04$ & $0.79(0.54-1.0)$ & $0.72(0.55-0.90)$ & $<0.001$ \\
\hline
\end{tabular}

JSW, joint space width; MCP, metacarpo-phalangeal joint; PIP, proximal interphalangeal joint; DIP, distal interphalangeal joint.

${ }^{a}$ Adjustments were made for age and gender.

${ }^{\mathrm{b}}$ All mean and adjusted mean differences were significant at the level $P<0.001$. 


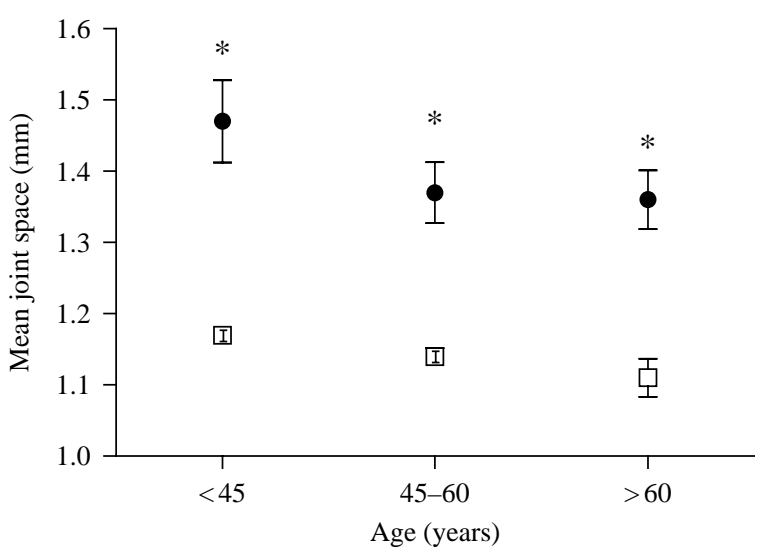

Figure 2 Increased mean (S.E.M.) of joint space widths of the hands in patients with acromegaly in three age groups compared with controls. Symbols represent the mean of all measured hand joint spaces (MCP, PIP, and DIP joints). Black circles, acromegalic patients; white squares, controls. ${ }^{*} P<0.001$.

was present between JSWs and age at diagnosis $(R=-0.28, P=0.009)$.

On linear regression analysis, pretreatment $\mathrm{GH}$ and IGF1 SDS were significantly associated with JSWs (mean of all joints and mean of MCP joints) with and without adjustments for age and gender (adjusted $\beta$ for pretreatment $\mathrm{GH}$ tertiles: $0.07(0.0-0.14)$ and for IGF1 tertiles: 0.09 (0.03-0.15); Table 2, Fig. 3). A younger age at diagnosis of acromegaly was associated with larger joint spaces also after adjustments for the duration of follow-up. The mean JSWs were not associated with different treatment modalities in this long-term controlled acromegaly cohort. Furthermore, JSWs were associated neither with current biochemical markers of disease activity in this biochemically controlled population nor with duration of remission or duration of follow-up.

\section{JSW and relation with complaints}

In general, there was no gender difference in the number of patients with self-reported pain in any joint of the hands. In individual joints, assessed by the structured diagram of 24 joints, female patients reported pain more frequently, i.e. in $16 \%$ of DIP joints, $21 \%$ of PIP joints, and $10 \%$ of MCP joints, compared with male patients who reported pain in $6.2 \%$ of DIP and PIP joints and $11.8 \%$ of MCP joints $(P<0.001$ for both DIP and PIP joints and $P=0.60$ (NS) for MCP joints). Joint pain was reported more frequently in joints with larger JSWs in male patients, when considering JSW in deciles per joint separately for gender $(\beta$ for male patient $0.15(0.05-0.26) P=0.004$, especially in the MCP joints, and for female patients $\beta 0.04(-0.02-$ 0.16) $P=0.184$ (NS); Fig. 4).

\section{Discussion}

The aim of the present study was to compare JSWs of hand joints in patients with long-term controlled acromegaly with a large control group, adjusted for age and gender. The data show that acromegalic patients have increased JSWs of their hand joints, indicating persistent cartilage hypertrophy despite longterm control of the disease. In addition, JSWs were related to $\mathrm{GH}$ and IGF1 concentrations at the time of initial diagnosis, but not to current concentrations of GH and IGF1, disease duration, or duration of follow-up. Finally, painful hand joints were wider than asymptomatic hand joints, especially in male patients. These observations characterize the late manifestations of well-controlled acromegaly in hand joints and indicate persistent, possibly irreversible, cartilage hypertrophy in these hand joints.

Arthropathy is an invalidating complication of active and well-controlled acromegaly (14). However, little is known about the clinical characteristics and progression or regression of acromegalic joint disease after induction of biochemical remission. Nonetheless, the prevalence of clinical and radiographic $O A$ is considerably increased in patients with long-term controlled acromegaly (4). A remarkable finding of our previous study was the combination of severe osteophytosis with normal or even widened joint spaces (4). In the present study, we document that the JSWs of the hand joints in acromegaly, especially the PIP and MCP joints, were clearly wider than those of controls. The increased joint spaces of the small hand joints are in accordance with the appearance of wide joint spaces on hip and knee radiographs of patients with long-term cure of acromegaly $(4,15)$. Therefore, joint space widening is a persistent feature of long-term controlled acromegaly in both small and large, weight- and nonweight-bearing joints.

Table 2 Linear regression analysis of factors influencing joint space width in metacarpo-phalangeal (MCP) joints and all hand joints in acromegaly patients. Data reported are unstandardized $\beta$ and confidence intervals. GH and IGF1 are categorized in groups according to tertiles. Pretreatment IGF1 s.D. and age are continuous variables. Proximal interphalangeal and distal interphalangeal joints were not influenced by markers of disease severity.

\begin{tabular}{lcc}
\hline & MCP joints $(n=8)$ & All joints $(n=24)$ \\
\hline Age (years) & $-0.009(-0.015$ to -0.003$)$ & $-0.005(-0.009$ to 0.000$)$ \\
Gender (M/F) & $-0.25(-0.38$ to -0.12$)$ & $-0.22(-0.32$ to -0.12$)$ \\
$\begin{array}{c}\text { Pretreatment } \\
\text { GH tertiles }(\mu \mathrm{g} / \mathrm{l})\end{array}$ & $0.09(0.03-0.184)^{\mathrm{a}}$ & $0.07(0.00-0.14)^{\mathrm{a}}$ \\
$\begin{array}{c}\text { Preoperative IGF1 } \\
\quad \text { (SDS) tertiles }\end{array}$ & $0.14(0.05-0.23)$ & $0.09(0.027-0.15)$ \\
$\begin{array}{c}\text { Pretreatment } \\
\text { IGF1 (SDS) }\end{array}$ & $0.018(0.02-0.035)$ & $0.012(0.001-0.023)$ \\
$\begin{array}{c}\text { Age at diagnosis } \\
\text { (years) }\end{array}$ & $\mathrm{NS}$ & $\mathrm{NS}$ \\
\hline
\end{tabular}

$n$, number of joints evaluated; NS, not significant.

${ }^{a}$ Adjustments for sex and present age. 

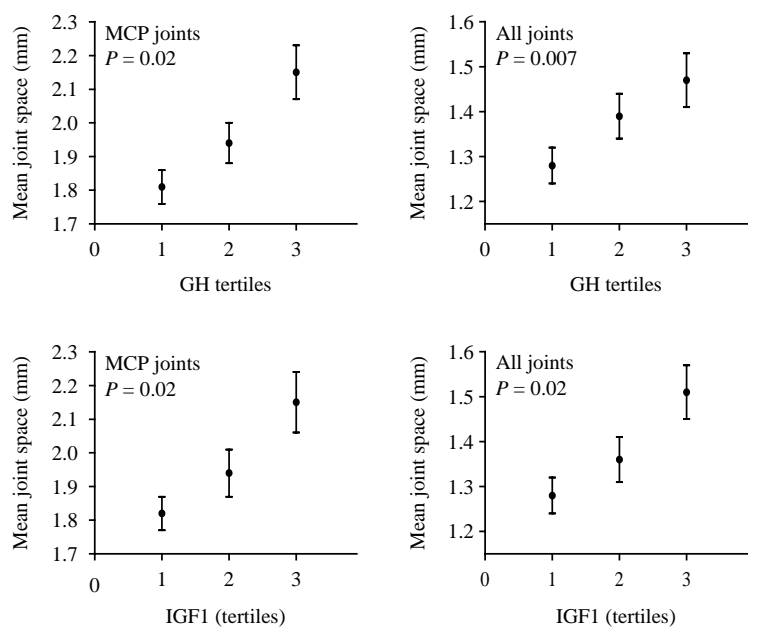

Figure 3 Mean joint space width (JSW; S.E.M.) increases with increasing pretreatment GH and IGF1 concentrations. Left, mean JSWs of MCP joints; right, mean JSWs of all 24 measured hand joints; upper panels, GH tertiles; lower panels, IGF1 tertiles.

Both GH and IGF1 act as growth factors for chondrocytes (16). In accordance with this notion, JSWs correlated positively with pretreatment GH and IGF1 concentrations. Age at diagnosis was negatively related to JSW, compatible with biochemically more active acromegaly at young age and/or, alternatively, with cartilage that is more responsive to GH-induced cartilage hypertrophy in younger subjects. JSWs were related to markers of disease severity at the time of initial diagnosis, but not to the duration of remission. This suggests that cartilage hypertrophy, induced during the active phase of the disease, is maintained in the long-term after cure of acromegaly. Apparently, the transient period of active acromegaly has resulted in a higher persistent set point of joint cartilage volume. Some short-term studies have suggested that cartilage hypertrophy may improve upon successful treatment of acromegaly $(17,18)$. However, in our study, the JSWs were still increased after long-term control of acromegaly, indicating persistent cartilage hypertrophy. In acromegalic patients, before treatment, joint spaces are more widened compared with values after early disease control, as indicated in the ultrasound studies by Colao et al. $(17,18)$. Therefore, in the present crosssectional study, we cannot differentiate between partial and complete irreversibility because pretreatment radiographs were not available for analysis. Additional follow-up measurements in acromegalic patients and studies in treatment-naïve patients are necessary to establish potential ongoing reversibility in the short term and during additional prolongation of follow-up.

We used a new semi-automated technique to quantify JSWs (5). This method is accurate and fast and can be used to determine joint space narrowing in patients with $\mathrm{OA}$ and is in good agreement with a semiquantitative scoring system of JSW (19). In the present study, we used a new technique to measure JSWs $(5,20)$. Previously, there was no validated method to assess joint space widening in the hands. By the use of validated qualitative scoring systems frequently used in primary OA, we experienced an important limitation of these scoring methods (i.e. Osteoarthritis Research Society (OARSI) scoring, Kellgren-Lawrence method), since widened joint spaces, a major feature of acromegalic arthropathy, are not included. With the present, new, and semi-automated method, we are the first to detect quantitative differences in JSWs in acromegalic patients compared with controls.

Moreover, within the group of acromegalic patients, this method was sensitive enough to relate JSWs to markers of disease severity of acromegaly. Further longitudinal studies are needed to assess whether this technique is sensitive enough to be used in clinical practice to detect ongoing GH/IGF1 stimulating effects on cartilage tissue, which would be very useful since we lack reliable biomarkers at present. The large number of abnormally wide joint spaces, which we defined as higher than the 95th percentile of controls, may have even been underestimated because of the somewhat lower age of the controls. Joints with increased JSWs were more frequently painful than joints within the range of normal, especially in male patients. In acromegalic patients, cartilage loss is probably not the main cause of pain, and therefore the reverse, i.e. cartilage hypertrophy, should be recognized as a risk factor for pain. Additional studies are needed to establish which factors are determinants of joint pains in these patients, such as osteophytosis, and instability of joints. Only $10 \%$ of the acromegalic patients used pain medication for joint complaints, on a chronic or on demand basis. The mean number of reported painful joints was not different
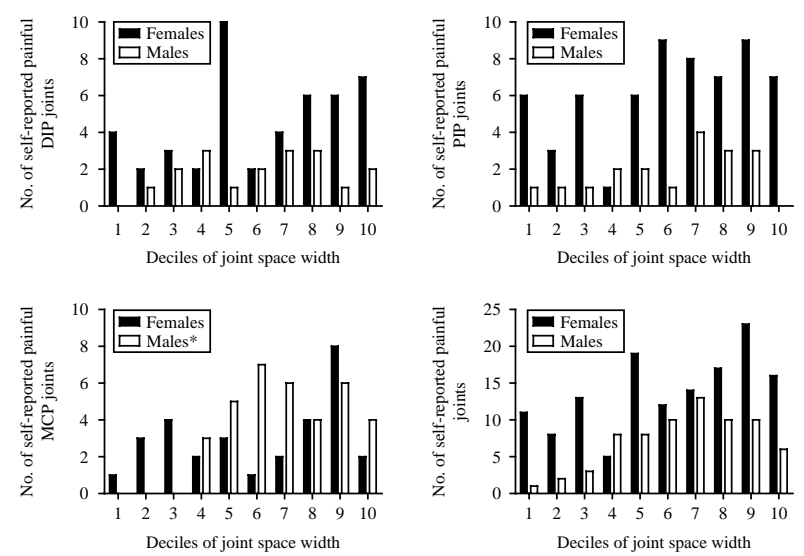

Figure 4 Bar chart of the distribution of self-reported pain in individual joints in 89 patients with acromegaly. Bars represent the number of painful joints in 89 acromegaly patients in the respective decile, for eight DIP, eight PIP, and eight MCP joints respectively (71.2 joints per decile evaluated). A higher decile reflects a higher joint space width (JSW). White bars, female patients; black bars, male patients. In men, more painful MCP joints are found in higher JSW deciles. ${ }^{\star} P<0.05$. 
between users of pain medication and non-users $(P=0.543)$. Therefore, we conclude that the use of pain medication does not affect our conclusions.

A limitation of this study is the possibility that the difference in age distribution between the various groups may have confounded the results to some extent. To correct for differences in age between patients and controls, we adjusted for age in all analyses. However, this may not necessarily control for the possible confounding effect of age because of the relatively small sample size. Nonetheless, in the present study, age had an effect on JSWs in patients with longterm control of acromegaly, but not in controls. Therefore, it is unlikely that the difference in age between controls and patients invalidates our conclusion with respect to the long-lasting effects of previous GH excess on JSWs.

In conclusion, the widening of joint spaces is a rather unique feature of acromegaly. Hand JSWs in acromegaly are positively related to GH and IGF 1 concentrations at the time of diagnosis and age at diagnosis, but not to the duration of follow-up, suggesting a dose-dependent and irreversible effect of previous $\mathrm{GH}$ excess on cartilage hypertrophy.

\section{Declaration of interest}

The authors declare that there is no conflict of interest that could be perceived as prejudicing the impartiality of the research reported.

\section{Funding}

This research did not receive any specific grant from any funding agency in the public, commercial or not-for-profit sector.

\section{References}

1 Melmed S. Medical progress: acromegaly. New England Journal of Medicine 2006355 2558-2573. (doi:10.1056/NEJMra062453)

2 Layton MW, Fudman EJ, Barkan A, Braunstein EM \& Fox IH. Acromegalic arthropathy. Characteristics and response to therapy. Arthritis and Rheumatism 198831 1022-1027. (doi:10.1002/art. 1780310813)

3 Biermasz NR, Pereira AM, Smit JW, Romijn JA \& Roelfsema F. Morbidity after long-term remission for acromegaly: persisting joint-related complaints cause reduced quality of life. Journal of Clinical Endocrinology and Metabolism 200590 2731-2739. (doi:10.1210/jc.2004-2297)

4 Wassenaar MJ, Biermasz NR, van Duinen N, van der Klaauw AA, Pereira AM, Roelfsema F, Smit JW, Kroon HM, Kloppenburg M \& Romijn JA. High prevalence of arthropathy, according to the definitions of radiological and clinical osteoarthritis, in patients with long-term cure of acromegaly: a case-control study. European Journal of Endocrinology 2009160 357-365. (doi:10.1530/EJE$08-0845)$

5 van 't Klooster R, Hendriks EA, Watt I, Kloppenburg M, Reiber JH \& Stoel BC. Automatic quantification of osteoarthritis in hand radiographs: validation of a new method to measure joint space width. Osteoarthritis and Cartilage 200816 18-25. (doi:10.1016/j. joca.2007.05.015)
6 Biermasz NR, Dekker FW, Pereira AM, van Thiel SW, Schutte PJ, van Dulken H, Romijn JA \& Roelfsema F. Determinants of survival in treated acromegaly in a single center: predictive value of serial insulin-like growth factor I measurements. Journal of Clinical Endocrinology and Metabolism 200489 2789-2796. (doi:10. 1210/jc.2003-032041)

7 van Aken J, van Bilsen JH, Allaart CF, Huizinga TW \& Breedveld FC. The Leiden Early Arthritis Clinic. Clinical and Experimental Rheumatology 200321 S100-S105.

8 Huetink K, Nelissen RGHH, Watt I, van Erkel AR \& Bloem JL. Localized development of knee osteoarthritis can be predicted from MR imaging findings a decade earlier. Radiology 2010256 536-546. (doi:10.1148/radiol.10090683)

9 Altman R, Alarcon G, Appelrouth D, Bloch D, Borenstein D, Brandt K, Brown C, Cooke TD, Daniel W \& Gray R. The American College of Rheumatology criteria for the classification and reporting of osteoarthritis of the hand. Arthritis and Rheumatism 199033 1601-1610. (doi:10.1002/art.1780331101)

10 Kellgren JH \& Lawrence JS. Radiological assessment of osteoarthrosis. Annals of the Rheumatic Diseases 195716 494-502. (doi:10.1136/ard.16.4.494)

11 Kellgren JH \& Lawrence JS. Osteo-arthrosis and disk degeneration in an urban population. Annals of the Rheumatic Diseases 195817 388-397. (doi:10.1136/ard.17.4.388)

12 Janssen YJ, Frolich M \& Roelfsema F. A low starting dose of genotropin in growth hormone-deficient adults. Journal of Clinical Endocrinology and Metabolism 199782 129-135. (doi:10.1210/ jc.82.1.129)

13 Cole TJ. The LMS method for constructing normalized growth standards. European Journal of Clinical Nutrition 1990 44 45-60.

14 Rikken B, van Doorn J, Ringeling A, Van den Brande JL, Massa G \& Wit JM. Plasma levels of insulin-like growth factor (IGF)-1, IGF1 and IGF-binding protein-3 in the evaluation of childhood growth hormone deficiency. Hormone Research $1998 \quad 50$ 166-176. (doi:10.1159/000023268)

15 Wassenaar MJ, Biermasz NR, Bijsterbosch J, Pereira AM, Meulenbelt I, Smit JW, Roelfsema F, Kroon HM, Romijn JA \& Kloppenburg M. Arthropathy in long-term cured acromegaly is characterised by osteophytes without joint space narrowing: a comparison with generalised osteoarthritis. Annals of the Rheumatic Diseases 201170 320-325. (doi:10.1136/ard.2010. 131698)

16 Giustina A, Mazziotti G \& Canalis E. Growth hormone, insulin-like growth factors, and the skeleton. Endocrine Reviews 200829 535-559. (doi:10.1210/er.2007-0036)

17 Colao A, Marzullo P, Vallone G, Giaccio A, Ferone D, Rossi E, Scarpa R, Smaltino F \& Lombardi G. Ultrasonographic evidence of joint thickening reversibility in acromegalic patients treated with lanreotide for 12 months. Clinical Endocrinology 199951 611-618. (doi:10.1046/j.1365-2265.1999.00851.x)

18 Colao A, Marzullo P, Vallone G, Marino V, Annecchino M, Ferone D, De Brasi D, Scarpa R, Oriente P \& Lombardi G. Reversibility of joint thickening in acromegalic patients: an ultrasonography study. Journal of Clinical Endocrinology and Metabolism 199883 2121-2125. (doi:10.1210/jc.83.6.2121)

19 Altman RD, Hochberg M, Murphy WA Jr, Wolfe F \& Lequesne M. Atlas of individual radiographic features in osteoarthritis. Osteoarthritis and Cartilage 19953 (Supplement A) 3-70.

20 Kwok WY, Bijsterbosch J, Malm SH, Biermasz NR, Huetink K, Nelissen RG, Meulenbelt I, Huizinga TW, van 't Klooster R, Stoel BC \& Kloppenburg M. Validity of joint space measurements in hand osteoarthritis. Osteoarthritis and Cartilage 201119 1349-1355. (doi:10.1016/j.joca.2011.08.011)

Received 9 September 2011

Revised version received 7 December 2011

Accepted 14 December 2011 